\title{
The Relationships of Personality and Cognitive Styles with Self-Reported Symptoms of Depression and Anxiety
}

\author{
Jonathan M. Sutton - Susan Mineka - Richard E. Zinbarg • \\ Michelle G. Craske · James W. Griffith - Raphael D. Rose • \\ Allison M. Waters $\cdot$ Maria Nazarian · Nilly Mor
}

Published online: 21 October 2010

(C) The Author(s) 2010. This article is published with open access at Springerlink.com

\begin{abstract}
Many studies have reported concurrent relationships between depressive symptoms and various personality, cognitive, and personality-cognitive vulnerabilities, but the degree of overlap among these vulnerabilities is unclear. Moreover, whereas most investigations of these vulnerabilities have focused on depression, their possible relationships with anxiety have not been adequately examined. The present study included 550 high school juniors and examined the cross-sectional relationships among neuroticism, negative inferential style, dysfunctional attitudes, sociotropy, and autonomy, with a wide range of anxiety and depressive symptoms, as well as the incremental validity of
\end{abstract}

J. M. Sutton $(\bowtie) \cdot$ S. Mineka $\cdot$ R. E. Zinbarg .

J. W. Griffith · N. Mor

Department of Psychology, Northwestern University,

2029 Sheridan Road, Swift Hall 102, Evanston, IL 60208, USA

e-mail: jonathansutton2009@u.northwestern.edu

R. E. Zinbarg

The Family Institute at Northwestern University,

Evanston, IL, USA

M. G. Craske - R. D. Rose - A. M. Waters - M. Nazarian

University of California at Los Angeles, Los Angeles, CA, USA

Present Address:

J. M. Sutton

The Edward Hines, Jr. VA Hospital, Hines, IL, USA

\section{J. W. Griffith}

The Feinberg School of Medicine, Northwestern University, Chicago, IL, USA

N. Mor

Hebrew University of Jerusalem, Jerusalem, Israel

A. M. Waters

The School of Psychology, Griffith University, Brisbane, Australia these different putative vulnerabilities when examined simultaneously. Correlational analyses revealed that all five vulnerabilities were significantly related to symptoms of both anxiety and depression. Whereas neuroticism accounted for significant unique variance in all symptom outcomes, individual cognitive and personality-cognitive vulnerabilities accounted for small and only sometimes statistically significant variance across outcomes. Importantly, however, for most outcomes the majority of symptom variance was accounted for by shared aspects of the vulnerabilities rather than unique aspects. Implications of these results for understanding cognitive and personality-cognitive vulnerabilities to depression and anxiety are discussed.

Keywords Neuroticism - Cognitive vulnerability · Incremental validity $\cdot$ Depression $\cdot$ Anxiety

\section{Introduction}

Psychological theories of vulnerabilities for depressive symptoms and disorders have proliferated for over 40 years. The list of potentially important vulnerabilities now includes personality traits such as neuroticism, personality-cognitive styles such as sociotropy and autonomy, and cognitive styles such as dysfunctional attitudes and negative inferential style. Significant concurrent relationships have been found between depressive symptoms and neuroticism (e.g., Klein et al. 2009), dysfunctional attitudes (reviewed in Clark and Beck 1999), negative inferential style (e.g., Haeffel et al. 2008), sociotropy (e.g., Robins et al. 1994), and autonomy (e.g., Zuroff 1994). Additional research has supported the validity of these constructs in prospective prediction of depressive symptoms and 
disorders (Alloy et al. 2006; Clark and Beck 1999; Clark et al. 1994; Klein et al. 2009).

The current study focuses on three important issues in this area of research that have received relatively little attention. First, although these theories of cognitive diatheses and personality-cognitive styles were originally hypothesized as vulnerabilities to depressive symptoms and disorders, little research has examined whether these are also diatheses for closely related (and highly comorbid) anxiety symptoms and disorders (e.g., Clark et al. 1994; Mineka et al. 1998). Second, relatively few investigations have examined these specific diatheses collectively to determine which are uniquely associated with depressive (or anxiety) symptoms and disorders. Finally, it is also not known whether these putatively more specific vulnerabilities for depression make unique contributions to symptoms above and beyond neuroticism $(\mathrm{N})$, which has already been well established as a predictor of anxiety and depression (e.g., Clark et al. 1994; Klein et al. 2009; Watson et al. 2005). Thus, the purpose of the present study was to examine these three issues in a cross-sectional sample of high school juniors participating in the Youth Emotion Project-an ongoing longitudinal study of common and specific risk factors for mood and anxiety symptoms and disorders.

\section{Cognitive Vulnerability Models for Depression} and Anxiety

Research on cognitive theories of depression has focused primarily on dysfunctional attitudes and negative inferential style. Dysfunctional attitudes were first described by Beck (e.g., 1967) whose theory posits that maladaptive or dysfunctional attitudes, beliefs, and assumptions about the self, world, and future predispose individuals to experience depression, particularly when these beliefs are activated by negative life events (e.g., Clark and Beck 1999). Abramson, Alloy, and colleagues have investigated a somewhat different cognitive diathesis-negative inferential style (e.g., Alloy et al. 2006). Negative inferential style refers to the tendency to interpret negative life events as leading to negative consequences, signifying fundamental flaws about the self, and having stable and global causes. Dysfunctional attitudes and inferential style are moderately correlated, with studies in unselected college samples reporting correlations between .4 and .5 (e.g., Hankin et al. 2005).

Although both of these cognitive styles were originally proposed as vulnerabilities for depression, researchers have questioned whether these styles might not also apply to anxiety, or to the overlap between anxiety and depression (e.g., Alloy et al. 1990; Mineka et al. 1995, 1998). First, substantial overlap has been documented between anxiety and depression at both the symptom and diagnostic levels (e.g., Clark and Watson 1991). Second, the proponents of these cognitive vulnerability theories have themselves cited the possibility of such relationships. For example, Haeffel et al. (2008) recently reviewed the literature on the Cognitive Style Questionnaire (CSQ) (e.g., Alloy et al. 2000) and cited a need for further investigation of its specificity to depression. Moreover, Clark and Beck (1999) noted that the scale used to measure their construct (DAS) is called the "Dysfunctional Attitudes Scale and not the Depressotypic or Depressogenic Attitudes Scale" (p. 137).

Several studies have reported significant relationships between anxiety symptoms and these putatively depressogenic vulnerabilities, although most studies have focused on only a few types of anxiety symptoms. For example, in a student sample, Hankin et al. (2004) reported moderate concurrent associations between both the DAS and the CSQ with general anxiety and anxious arousal symptoms assessed by the Mood and Anxiety Symptom Questionnaire (Watson et al. 1995a). Hankin (2008a) also found moderate correlations between inferential style and anxious arousal symptoms concurrently and longitudinally in a sample of sixth to tenth graders. Finally, Oliver et al. (2007) reported small to moderate associations between both the DAS and the CSQ with anxiety symptoms on the Beck Anxiety Inventory (Beck et al. 1988). The present study extends these associations to a wider range of anxiety symptomatology.

\section{Personality-Cognitive Styles and Depression} and Anxiety

Sociotropy and autonomy are two personality-cognitive style constructs that have been hypothesized to confer vulnerability to depression (e.g., Clark and Beck 1999). Individuals scoring highly on sociotropy are described as basing their self-worth on the closeness and security of social relations with others, whereas those scoring highly on autonomy are described as basing their self-worth on achievement, independence, and control. These two personality-cognitive styles are partially independent, with the two constructs correlating only .20 in two college student samples (Robins et al. 1994).

Although several studies have reported significant main effects of sociotropy and autonomy on concurrent selfreported depression (e.g., Frewen and Dozois 2006; Robins et al. 1994), there has been only limited investigation into whether they are also predictors of anxiety symptoms. For example, Alford and Gerrity (1995) reported significant concurrent associations between sociotropy (but not autonomy) and symptoms of both depression and anxiety. However, Fresco et al. (2001) found concurrent associations between sociotropy and anxiety (but not depressive) symptoms, whereas autonomy related only to depressive symptoms. Clearly more research is needed on this topic before drawing any firm conclusions as the empirical work to date is quite limited in depth and scope. 


\section{Neuroticism}

The present study sought to compare the incremental validity of these putatively more specific diatheses to the broader personality trait of $\mathrm{N}$ which has been defined as a relatively stable trait tapping one's sensitivity to negative stimuli and the tendency to experience negative mood states (e.g., Clark et al. 1994; Eysenck and Eysenck 1975). In a widely cited early review, Clark et al. (1994) concluded that high $\mathrm{N}$ confers increased risk for both anxiety and depression. Over the past 15 years, a significant body of additional research has further documented these associations (see Klein et al. 2009; Watson et al. 2005, for recent reviews).

\section{Incremental Validity of Predictors}

As noted, one objective of the present study was to examine the degree to which cognitive style and personality-cognitive vulnerabilities make unique contributions to symptoms of depression and/or anxiety. The present investigation also assessed the incremental validity of these other vulnerabilities to associations with depressive and anxiety symptoms beyond the variance accounted for by $\mathrm{N}$ and, conversely, the incremental validity of $\mathrm{N}$ beyond these other vulnerabilities (see Paunonen 1998). A number of studies have examined unique and overlapping components of these vulnerabilities (e.g., Dunkley et al. 1997; Hankin et al. 2004, 2005; Mongrain and Blackburn 2005; Oliver et al. 2007). For example, using a longitudinal design, Hankin et al. (2004, Study 2) found that when the CSQ and the DAS were examined simultaneously in the prediction of depressive symptoms and depressive disorder, neither main effects, nor diathesis-stress interactions (entered on a subsequent step), were uniquely significant for either cognitive style. Thus, they concluded that associations with depression are found in the overlapping components of those cognitive vulnerabilities. Dunkley et al. (1997) examined the incremental validity of sociotropy, autonomy, and $\mathrm{N}$ in associations with depressive symptoms using a cross-sectional design. They reported that when $\mathrm{N}$ was entered into equations predicting depressive symptoms, solitude (a facet of autonomy) in men, and sociotropy in women, were no longer significant predictors of symptoms. They concluded that $\mathrm{N}$ might account for much of the association between sociotropy, autonomy, and general distress (see also Zuroff et al. 2004a).

To our knowledge, only two other studies have examined all five of these vulnerabilities simultaneously. Mongrain and Blackburn (2005) examined them in a sample of 97 graduate students who each had a prior history of depressive disorder. They reported that both attributional style and dysfunctional attitudes were uniquely associated with the number of prior depressive episodes above and beyond neuroticism, sociotropy, and autonomy; none of the latter was uniquely significant. Analyses predicting recurrence of depression over the following 16 months demonstrated unique contributions from attributional style and autonomy. No significant relationships were found between cognitive styles and current or past anxiety diagnoses. Our study differs in several important ways from the Mongrain and Blackburn study. First, whereas our study investigates a large community-based adolescent sample, Mongrain and Blackburn had a highly selected sample of graduate students with a history of mood disorder, raising serious questions about the generalizability of their results. Second, our analyses examine five different types of anxiety symptoms separately versus combining them into a single dimension because the strength of associations between these vulnerabilities and anxiety may well differ among types of anxiety (see Mineka et al. 1995). Finally, our study $(n>500)$ has substantially greater power to detect small unique effects than did Mongrain and Blackburn $(n=97)$.

A second investigation was also conducted on the YEP data set (Zinbarg et al. 2010) with objectives related to those of the present investigation. Using logistic regression, Zinbarg et al. examined the incremental validity of cognitive and personality-cognitive vulnerabilities and $\mathrm{N}$ in their relationships with past diagnoses of anxiety and depressive disorders in the same sample of adolescents. Using current depression and/or anxiety symptoms as covariates, Zinbarg et al. (2010) reported that $\mathrm{N}$ was uniquely associated with past mood disorders (although only for men) and past anxiety disorders, while no other vulnerability made a unique contribution.

In contrast to the study by Zinbarg et al. (2010) which examined only past diagnoses, the present study focuses on current symptoms. This focus on current symptoms of anxiety and depression is important, particularly if the results differ from those found with diagnoses. Such symptom level analyses are consistent with a large number of studies examining cognitive and personality-cognitive vulnerabilities using dimensional models of psychopathology. Further, the present study evaluated specific symptom clusters for different types of mood and anxiety symptoms whereas Zinbarg et al. collapsed outcomes into depressive and/or anxiety disorders because of limited sample sizes for some individual disorders. Moreover, both clinical and sub-clinical levels of depressive symptoms are associated with psychosocial dysfunction (e.g., Lewinsohn et al. 2000) and sub-clinical depressive and anxiety symptoms have been associated with later risk for mood and anxiety disorders in numerous studies (e.g., Fergusson et al. 2005; Murphy et al. 1989; Pine et al. 1999). Thus, even if the relationships between certain putative vulnerabilities and past diagnoses were not significant, a 
significant association between these same vulnerabilities and current symptoms could still indicate risk for later disorder.

\section{Objectives}

The present study had three objectives. First, we examined whether cognitive and personality-cognitive styles theorized to be related to depression were also significantly related to symptoms of different types of anxiety. Two patterns of results could be hypothesized based on past research and theory. A specificity hypothesis would suggest that whereas all five vulnerabilities should be significantly associated with depression, only $\mathrm{N}$ should be significantly related to anxiety symptoms. Alternatively, a nonspecificity hypothesis suggests that vulnerabilities for depression may be vulnerabilities for at least some types of anxiety as well.

The second and third objectives examined the incremental validity of the cognitive style, personality-cognitive style, and neuroticism vulnerabilities for each of eight outcomes: two depression scales, a depression symptom composite, and five scales measuring different types of anxiety symptoms. These comparisons initially examined just the four specific vulnerabilities and subsequently included the broader neuroticism vulnerability. If individual vulnerabilities were significant unique predictors of symptom outcomes, it would indicate that meaningful variance was accounted for by the non-overlapping components of each diathesis. Alternatively, if the group of predictors as a whole accounted for significant variance in depressive or anxiety symptoms, but the individual predictors did not, such findings would support the hypothesis that the overlapping aspects of the vulnerabilities were the key to predicting symptoms. Thus, our second and third objectives focused on the degree to which overlapping versus unique aspects of these vulnerabilities were associated with current mood and anxiety symptoms.

\section{Method}

\section{Participants}

The present investigation focused on data collected at the initial assessment for three cohorts of adolescents recruited in three consecutive academic years beginning in winter of 2003 as part of a prospective study (see also Zinbarg et al. 2010). At the time of study recruitment, all students were in their junior year at two large public high schools, one in suburban Chicago and one in suburban Los Angeles. After receiving parental consent and student assent, Ps completed a screening questionnaire to assess $\mathrm{N}$ : the Eysenck
Personality Questionnaire (EPQ-R-N; Eysenck et al. 1985), which was used to stratify them based on their level of neuroticism. Ps were paid ten dollars for their participation in the screening phase.

In total, 1,976 students filled out the EPQ-R-N ${ }^{1}$ and were eligible for the study. Students scoring in the upper $33 \%$ on the neuroticism questionnaire were overrecruited relative to middle and lower-third Ps. Of the 1,976 students who completed the EPQ-R-N, 1,269 were invited to participate in the study, and 627 students consented to, and participated in, our Time 1 data collection across both sites ( $\mathrm{N}=305$ at Northwestern University; $\mathrm{N}=322$ at UCLA). Seventy-seven cases were excluded from the present analyses because of missing data on one or more of the self-report measures (described below) of cognitive styles, personality-cognitive styles, $\mathrm{N}$, and/or symptoms of psychopathology.

The final sample for the present analyses was 550. At the Time 1 assessment, Ps had a mean age of 16.9 $(S D=.4)$ and were predominantly female: $(n=381$, $69 \%)$. This gender difference was not intended but was due in part to women both being more likely to complete the screening questionnaire and to enroll in the study if invited. In addition, as expected based on prior findings (e.g., Costa et al. 2001), women $(M=11.1, S D=4.6)$ scored significantly higher than men $(M=9.2, S D=4.6)$ on the EPQ$\mathrm{R}-\mathrm{N}, t(1,962)=8.95, P<.01$. Based on the EPQ-R-N, Ps were classified as being high- $(n=326 ; 59 \%)$, medium$(n=126 ; 23 \%)$ or low-EPQ-R-N $(n=98 ; 18 \%)$ scorers. The ethnic composition of the sample of 550 Ps was as follows: African American, 12.0\%; Asian American or Pacific Islander, 5.2\%; Caucasian, 48.7\%; Hispanic or Latin American, 14.7\%; other or mixed ethnicity, $19.3 \%$.

\section{Procedure}

At Time 1, Ps completed interviews assessing life stress and diagnoses of Axis I disorders (see Zinbarg et al. 2010). Either at the same session or at a session shortly thereafter, Ps filled out a battery of questionnaires including vulnerability measures and symptom outcomes. Ps were paid forty dollars for completing the interviews and questionnaires.

Measure of Neuroticism

\section{International Personality Item Pool-NEO-PI-R}

The $\mathrm{N}$ scale from the IPIP-NEO-PI-R consists of 60 items and closely corresponds with the N scale from the NEO-PI-

\footnotetext{
${ }^{1}$ Because the EPQ-R is not a component of any analyses in the present study, the reader is referred to Mor et al. (2008) for psychometric information regarding that scale in our study.
} 
R (IPIP 2001; Costa and McCrae 1985). ${ }^{2}$ All items are rated on a 1-5 Likert-type scale from "very inaccurate" to "very accurate." Approximately half of the items are reverse-keyed. Each of the six subscales of the IPIP-N scale has been found to have adequate internal consistency ( $\alpha>$.77), and Goldberg (1999) reported that the total score from the NEO-PI-R was highly correlated with the IPIP-N: $(r=.93)$. The full scale measure administered at Time 1 had good internal consistency $(\alpha=.95)$; coefficient omega $_{\text {hierachical }}$ (Zinbarg et al. 2006) was .86.

Some of the strong association between $\mathrm{N}$ and symptoms of anxiety and depression may well be due to overlapping content in the scales measuring those constructs. In order to address possible criterion contamination between $\mathrm{N}$ and the symptom measures, we removed 25 items from the scale. ${ }^{3}$ The 35 -item scale also had good internal consistency $(\alpha=.89)$.

\section{Measures of Cognitive Vulnerability}

\section{Cognitive Style Questionnaire (CSQ)}

Ps' inferential style for hypothetical negative events was measured by the Cognitive Style Questionnaire (see Alloy et al. 2000; Haeffel et al. 2008). Only the 12 negative events were assessed in this study. Each hypothetical event on the CSQ is rated on five scales. Scores on each scale range from 1 to 7 with higher scores representing a more negative inferential style. The first three scales are measures of pessimistic attributional style and contain the dimensions of internality, stability, and globality (Peterson et al. 1982). In addition, Ps are asked to rate the likelihood of other negative consequences stemming from the hypothetical event and the negative implications about the self. A composite score for negative inferential style was formed by combining the ratings on four of the scales: globality, stability, consequences, and implications about the self. Alloy et al. (2000) demonstrated that this four scale composite has strong internal consistency $(\alpha=.88)$ as was the case in this study $(\alpha=.89)$.

\footnotetext{
${ }^{2}$ In the present study we focused on results using a single measure of neuroticism. Other articles derived from this project have modeled neuroticism based on four neuroticism scales (e.g., see Griffith et al. 2010a; Zinbarg et al., 2010). Due to space constraints and reviewer feedback specific to the present study, we report results using this single modified neuroticism scale. Regression analyses involving neuroticism were also conducted incorporating the neuroticism composite used in other articles by our research team. These results are available from the first author and they are largely similar to the results presented in the text.

3 The following 25 -items were removed from the IPIP-NEO-PI-R: 2, $5,6,7,9,10,11,12,14,19,21,22,23,24,25,27,29,30,33,36,39$, $51,54,58$, and 60 .
}

Dysfunctional Attitudes Scale (DAS)

Another measure of cognitive vulnerability was the Dysfunctional Attitudes Scale (DAS; Weissman and Beck 1978). The present study used the standard 40 items from the original version, plus an additional 24 age- and studentappropriate items that were added in the Cognitive Vulnerability to Depression project (CVD; Alloy et al. 2000). Ps endorsed items on a 7-point scale from "totally agree" to "totally disagree." Most items are reverse coded, such that the scale taps a participant's agreement with negative self-statements. Alloy et al. reported an alpha coefficient of .90 for their screening sample. Coefficient alpha for the full 64 items was high in this study $(\alpha=.95)$.

Measure of Personality-Cognitive Style

Personal Style Inventory (PSI-II)

Sociotropy and autonomy were measured using the revised Personal Style Inventory (PSI-II; Robins et al. 1994). The measure consists of two scales each containing 24 items. All items are endorsed on a 1-6 scale from "strongly disagree" to "strongly agree." Both sociotropy $(\alpha=.90)$ and autonomy ( $\alpha=.85)$ showed good internal consistency.

Measures of Depressive and Anxiety Symptomatology

Mood and Anxiety Symptom Questionnaire (MASQ)

One measure of symptoms was the Mood and Anxiety Symptom Questionnaire (Watson et al. 1995a). The MASQ consists of 90 items that the participant rates on a 1-5 scale from "not at all" to "extremely." The present study focused on four subscales: General Distress: Anxiety (11 items); General Distress: Depression (12 items); Anxious Arousal (17 items); and Anhedonic Depression (22 items comprised of 8 loss of interest items and 14 reverse-scored high positive affect items). Each of the scales has demonstrated good internal consistency $(\alpha>.8)$ in student, adult, and patient samples (Watson et al. 1995b). Furthermore, the specific symptom scales, Anhedonic Depression and Anxious Arousal, have demonstrated excellent convergent and discriminant validity (Watson et al. 1995b). In this study, each of the four scales had good internal consistency $(\alpha>.84)$.

\section{Inventory to Diagnose Depression (IDD)}

The IDD (Zimmerman et al. 1986) is a 22-item self-report measure that can be used to approximate diagnoses of major depressive disorder, although we used it as a symptom measure rather than a diagnostic one. Zimmerman et al. 
(1986) reported convergent validity with other interview and self-report measures, and high internal consistency $(\alpha=.92)$ in their sample of depressed and nondepressed Ps. The present study removed one item assessing suicidal/death thoughts because of IRB concerns. Coefficient alpha for the 21 -item IDD in the present sample was good $(\alpha=.88)$.

\section{Social Phobia Scale (SPS)}

This study used the 13-item version of the SPS (Mattick and Clarke 1998), which assesses symptoms of social phobia from the self-consciousness subscale. Zinbarg and Barlow (1996) reported that this 13-item version has strong internal consistency ( $\alpha=.92$ ) and construct validity, and is more factorially homogeneous than the full SPS. In the present study, the internal consistency for this scale was $\operatorname{good}(\alpha=.89)$.

\section{Situational Fears Questionnaire (SFQ)}

A 22-item measure assessing fears was adapted from the Albany Panic and Phobia Questionnaire (Rapee et al. 1995). The scale contains 11 items that measure interoceptive fears and 11 items that assess agoraphobic situations. These two scales correlate highly (Zinbarg and Barlow 1996) and were combined to yield a total scale score. In the current study, internal consistency was good $(\alpha=.89)$.

\section{Fear Survey Schedule (FSS)}

A 10-item scale was derived from the larger Fear Survey Schedule-II (Geer 1965). Items from three subscales identified by Zinbarg and Barlow (1996) were included: blood/injury, heights, and animals. Because the present study sought to operationalize fear on a continuum rather than for diagnostic purposes, the 10 items were summed to form a single score $(\alpha=.83)$.

\section{Preparation for Analyses}

A depressive symptom composite was formed consisting of the IDD, MASQ-General Depression, and MASQ-Anhedonic Depression scales. Scores from each of the three scales were standardized and averaged $(\alpha=.84)$. One value was clearly separated from the rest of the distribution and was recoded to reduce its influence (Tabachnick and Fidell 2001).

\section{Plan for Analyses}

The first objective of the study was to determine whether depressogenic vulnerabilities were also related to anxiety.
Accordingly, we examined bivariate associations among the four specific vulnerabilities and the anxiety and depression symptom measures. The second objective of the study was to examine the overlapping and unique associations of cognitive and personality-cognitive vulnerabilities with the symptom measures of anxiety and depression. A set of eight multiple linear regressions was conducted with the five anxiety symptom scales, two MASQ depression scales, and the depression composite as dependent variables.

A similar analytic approach was taken to address the third study objective which was to examine whether the more specific cognitive and personality-cognitive style vulnerabilities made unique contributions to symptoms of anxiety and depression beyond $\mathrm{N}$, and vice versa. ${ }^{4} \mathrm{~A}$ set of eight multiple linear regressions was conducted. For linear regression analyses, partial regression coefficients were interpreted as significant only if the overall model was significant at $P<.05$ and the parameter estimate was also significant at $P<.05$.

\section{Results}

Bivariate Relationships Between Vulnerabilities and Symptoms

Mean-item scores and standard deviations for predictor and outcome variables are shown in Table 1. Neuroticism, sociotropy, autonomy, dysfunctional attitudes, and inferential style were all significantly correlated ( $r$ 's ranging from .42 to $.58, P$ ' $s<.01$ ). Furthermore, each of the vulnerabilities was significantly associated with the depression composite (r's ranging from .46 to .67, $P$ 's $<.01$ ) and with both general and anhedonic depressive symptoms ( $r$ 's ranging from .28 to .63). All correlations between vulnerabilities and anxiety symptoms were also statistically significant $(P<.01)$. Correlations ranged from .13 for the association between specific fears and DAS to .54 for the association between social phobia symptoms and $\mathrm{N}$.

\footnotetext{
${ }^{4}$ There may be some question as to whether the DAS, which has been divided into subscales focused on achievement and social valuation in prior studies, might be undermined in the analyses by the inclusion of sociotropy and autonomy. The theoretical rationale for including all of the vulnerabilities in this study was to help develop parsimonious models. Notwithstanding, some supplementary analyses were conducted looking only at N, CSQ, and DAS. DAS accounted for no more than $2 \%$ of unique variance in any of these regression analyses.
} 
Table 1 Descriptive statistics and correlations among personality, cognitive styles, personality-cognitive styles, and symptoms of depression and anxiety

\begin{tabular}{|c|c|c|c|c|c|c|c|c|c|c|c|c|c|c|}
\hline Variable & 1 & 2 & 3 & 4 & 5 & 6 & 7 & 8 & 9 & 10 & 11 & 12 & 13 & 14 \\
\hline \multicolumn{15}{|l|}{ 1. $\mathrm{N}$} \\
\hline 2. CSQ & .50 & & & & & & & & & & & & & \\
\hline 3. DAS & .58 & .50 & & & & & & & & & & & & \\
\hline 4. SOC & .56 & .48 & .53 & & & & & & & & & & & \\
\hline 5. AUT & .50 & .42 & .50 & .46 & & & & & & & & & & \\
\hline 6. IDD & .56 & .43 & .44 & .39 & .41 & & & & & & & & & \\
\hline 7. MASQ-GD & .63 & .50 & .50 & .52 & .49 & .72 & & & & & & & & \\
\hline 8. MASQ-AD & .56 & .38 & .44 & .28 & .44 & .56 & .61 & & & & & & & \\
\hline 9. DEPCOMP & .67 & .50 & .53 & .46 & .51 & .88 & .90 & .83 & & & & & & \\
\hline 10. MASQ-GA & .52 & .39 & .35 & .38 & .39 & .58 & .72 & .38 & .65 & & & & & \\
\hline 11. MASQ-AA & .39 & .28 & .30 & .28 & .35 & .53 & .63 & .34 & .58 & .77 & & & & \\
\hline 12. SPS & .54 & .39 & .39 & .44 & .42 & .47 & .50 & .37 & .51 & .40 & .41 & & & \\
\hline 13. SFQ & .34 & .24 & .23 & .29 & .23 & .36 & .34 & .20 & .34 & .34 & .34 & .54 & & \\
\hline 14. FSS & .33 & .19 & .13 & .24 & .17 & .21 & .23 & .13 & .22 & .21 & .19 & .38 & .47 & \\
\hline Mean-Item & 2.78 & 3.45 & 3.12 & 3.65 & 3.42 & 0.53 & 2.11 & 2.68 & - & 1.81 & 1.51 & 0.97 & 0.95 & 1.78 \\
\hline SD & 0.54 & 1.00 & 0.84 & 0.77 & 0.65 & 0.43 & 0.87 & .65 & - & 0.65 & 0.57 & 0.73 & 0.89 & 1.08 \\
\hline
\end{tabular}

All correlations are significant at $P<.01$

$N$ neuroticism measure (35-item IPIP), CSQ cognitive style questionnaire composite, DAS dysfunctional attitudes scale, SOC Sociotropy, $A U T$ autonomy, $I D D$ inventory to diagnose depression, $M A S Q-G D$ mood and anxiety symptom questionnaire general depression, $M A S Q$ $A D$ anhedonic depression, DEPCOMP depression composite, MASQ-GA general anxiety, MASQ-AA anxious arousal, SPS social phobia scale, $S F Q$ situational fears questionnaire, FSS fear survey schedule

Specific Vulnerabilities and Symptoms of Depression and Anxiety

Regression analyses examining the four specific vulnerabilities are presented in Table 2. The four specific vulnerabilities accounted for about $40 \%$ of the variance for both the depression composite and general depressive symptoms with each vulnerability making a statistically significant unique contribution between 1 and 4\%. For anhedonic depression symptoms, the four vulnerabilities accounted for $28 \%$ of the variance and the CSQ, DAS, and autonomy contributed 2, 4, and $5 \%$ of unique variance respectively. For general anxiety symptoms, the vulnerabilities together accounted for $24 \%$ of the variance with CSQ, sociotropy, and autonomy, each contributing 2-3\% of unique variance. For anxious arousal symptoms, the set of four vulnerabilities accounted for $16 \%$ of the variance with the greatest unique contribution (4\%) made by autonomy. For social phobia symptoms, the four vulnerabilities accounted for $28 \%$ of the variance with CSQ, DAS, sociotropy, and autonomy uniquely contributing 1-3\% of variance. Relatively smaller amounts of total variance were accounted for in agoraphobic/interoceptive fears $\left(R^{2}=.10\right)$ and specific fears $\left(R^{2}=.07\right)$. Sociotropy made small, but significant contributions to both of the latter symptom sets, and CSQ accounted for $1 \%$ of unique variance in specific fears. In sum, these vulnerabilities contributed significantly as a group to all five anxiety symptom scales as well as to depressive symptoms. Notably, each of the vulnerabilities accounted for a small amount of unique variance in at least two of the anxiety symptom scales.

\section{Overlap of the Specific Vulnerabilities}

A related objective of these analyses was to compare the shared versus unique contributions of the four specific vulnerabilities in their associations with symptoms of anxiety and depression. Importantly, the magnitude of the unique contributions was relatively small compared to the overall shared variance (see right side of Table 2). Indeed, approximately $70 \%$ of the variance accounted for in the depression composite and general depressive symptoms derived from factors shared by the vulnerabilities. Very similar results were found for social phobia, general anxiety, agoraphobic/interoceptive fears, and anxious arousal symptoms. Unique variance played a somewhat larger role in anhedonic depressive symptoms (39\%) and specific fears (51\%). Substantial evidence then suggests that what is shared among these cognitive and personality-cognitive vulnerabilities comprises a significant proportion of their relationships with symptoms. 


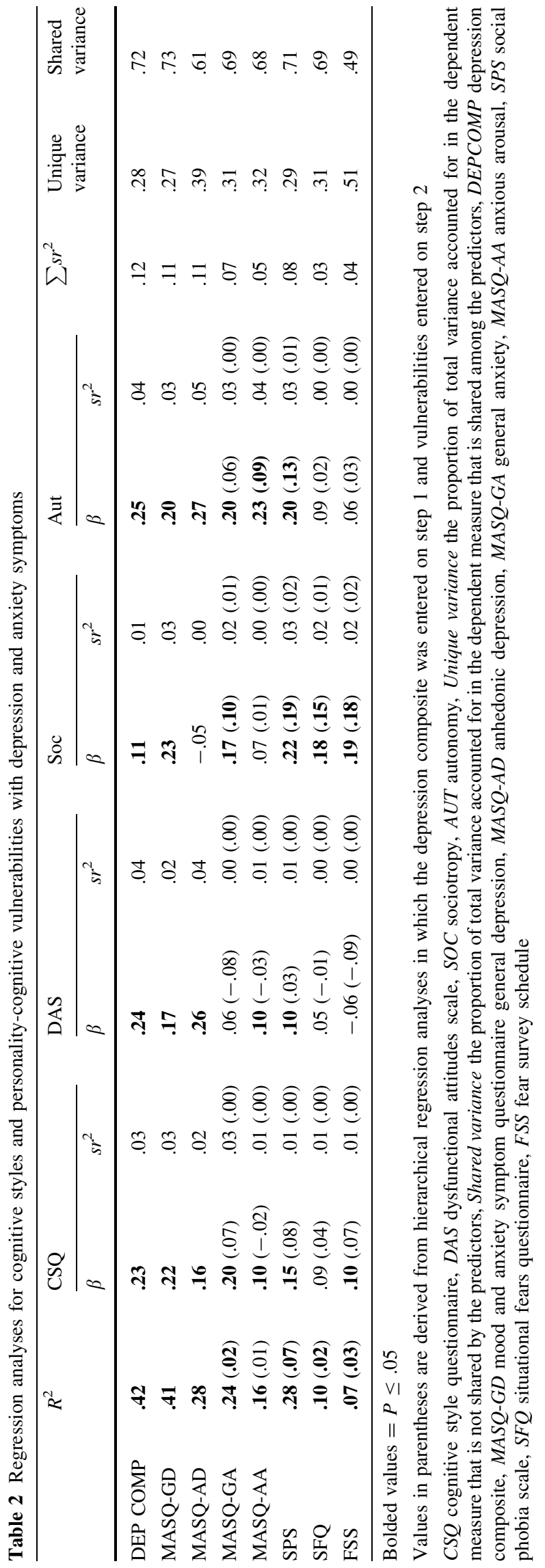

Incremental Validity Analyses Examining Specific Vulnerabilities and $\mathrm{N}$

A third objective of the study was to examine the incremental validity of the more narrow vulnerabilities as compared to $\mathrm{N}$ (see Table 3). As a group, the five vulnerabilities accounted for about $50 \%$ of variance in the depression composite and general depression symptoms. The largest unique contribution was made by $\mathrm{N}$ which accounted for nearly $11 \%$ of unique variance in the composite and $7 \%$ for general depressive symptoms. For anhedonic depression symptoms, the five vulnerabilities accounted for about $37 \%$ of the variance, with $\mathrm{N}$ accounting for $9 \%$ of unique variance. Across the three depression measures, with two exceptions, all four of the more narrow vulnerabilities contributed significant unique variance ranging from 1 to $2 \%$. Thirty-one percent of the variance in general anxiety symptoms was accounted for by the five vulnerabilities. Neuroticism again made the largest unique contribution $\left(s r^{2}=.07\right)$, with CSQ and autonomy each making very small, but statistically significant additions. As a group, the five vulnerabilities accounted for $19 \%$ of the variance in symptoms of anxious arousal. The largest unique contributions came from neuroticism $\left(s r^{2}=.03\right)$ and autonomy $\left(s r^{2}=.02\right)$; the other three vulnerabilities did not make significant unique contributions. For social phobia symptoms, 34\% of the variance was accounted for by the five vulnerabilities, with neuroticism $\left(s r^{2}=.06\right)$ providing the largest contribution, and CSQ, sociotropy and autonomy each contributing $1 \%$ of unique variance. The vulnerabilities accounted for approximately $13 \%$ of the variance in agoraphobic/interoceptive fears. Neuroticism and sociotropy made significant unique contributions of 3 and $1 \%$ of variance, respectively. Finally, all five vulnerabilities accounted for about $13 \%$ of the variance in specific fears. Neuroticism made the largest unique contribution of about $6 \%$ of variance while DAS and sociotropy each contributed $1 \%$ of unique variance.

In sum, neuroticism made significant unique contributions to all depression and anxiety symptom measures, and made the largest unique contribution for all outcomes. Inferential style contributed uniquely only to depressive symptoms, general anxiety, and social fears. Dysfunctional attitudes contributed uniquely only to the depression composite, anhedonic depressive symptoms, and specific fears (an inverse relationship). Sociotropy made a significant unique contribution to general depressive symptoms, anhedonic symptoms (an inverse relationship), social fears, agoraphobic/interoceptive fears, and specific fears. Finally, autonomy made significant unique contributions to symptoms of depression, anxious arousal, general anxiety, and social phobia. 


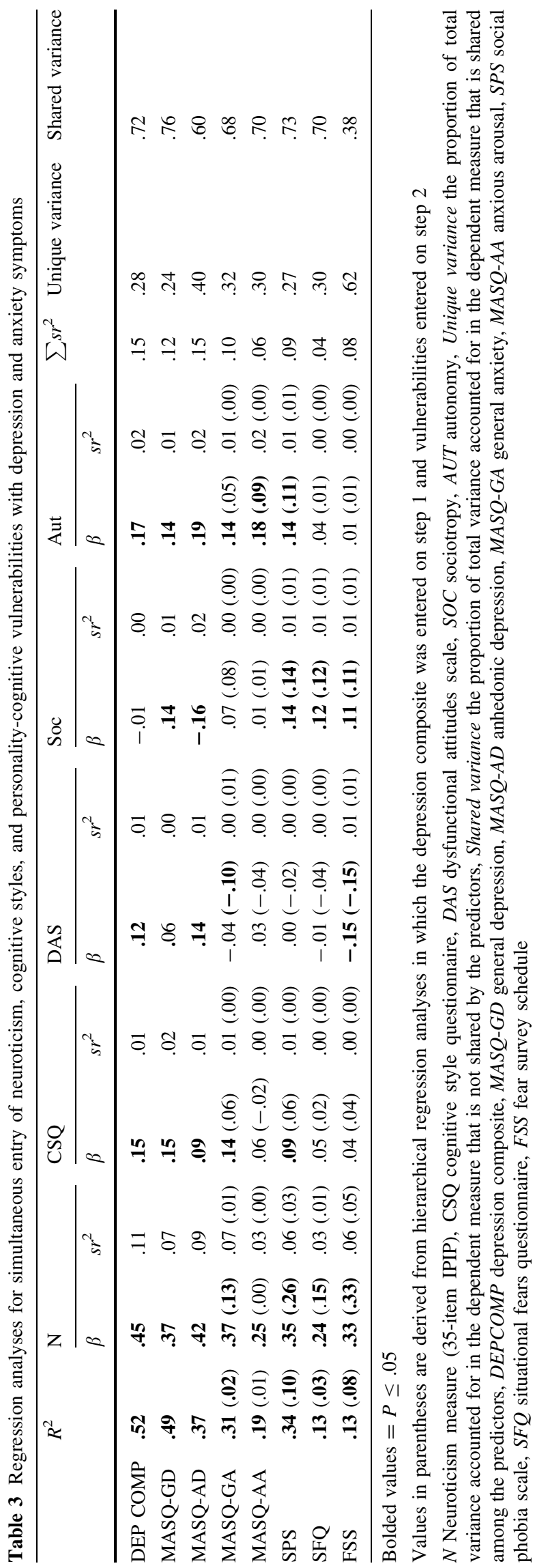

Overlap of the Five Vulnerabilities

For all symptom outcomes with the exception of anhedonic depression and specific fears, at least two-thirds of the variance accounted for by the vulnerabilities was shared among the vulnerabilities (range 68-76\%; see last column of Table 3). For anhedonic depression approximately $60 \%$ of the variance accounted for by the vulnerabilities was associated with shared factors. For specific fears, about $60 \%$ of the variance was accounted for by unique factors. Notably, $\mathrm{N}$ accounted for almost $75 \%$ of the unique variance in specific fears.

Analyses Predicting Anxiety Symptoms Using a Depression Symptom Covariate

To examine whether the significant relationships between these vulnerabilities and anxiety outcomes were fully explained by shared variance with depression, hierarchical regression analyses were conducted for all anxiety symptom outcomes: The depression symptom composite was entered on step 1 and the vulnerabilities were entered on step 2. These results are presented within parentheses in Tables 2 and 3. Notably, the overall contribution of the vulnerabilities was substantially decreased relative to the original analyses; however, the group of vulnerabilities still accounted for significant variance for all outcomes except anxious arousal. These results suggest that the overlap of depression and anxiety symptoms largely, but not completely, explains the relationship between the vulnerabilities and anxiety outcomes. A second notable finding was that sociotropy (see Table 2) and N (see Table 3) were significant predictors of four anxiety outcomes beyond the depression composite, whereas CSQ was no longer significantly uniquely associated with any anxiety outcome.

\section{Discussion}

The present study sought to extend the limited amount of research to date on the relationship between symptoms of anxiety and previously studied vulnerabilities for depression as well as to examine the unique predictive power of specific diatheses. We also examined the incremental validity of the more narrowly defined specific vulnerabilities and $\mathrm{N}$.

Relationships Between Vulnerabilities and Symptoms of Anxiety

One major finding of the present study was the substantial association between a variety of anxiety symptoms and 
dysfunctional attitudes, negative inferential style, autonomy, and sociotropy. Substantial concurrent associations were found between the four specific vulnerabilities and symptoms of social phobia ( $r^{2}$ range $\left.15-19 \%\right)$, general anxiety $\left(r^{2}\right.$ range $\left.12-15 \%\right)$, and anxious arousal $\left(r^{2}\right.$ range $8-12 \%)$. Although smaller in absolute size than associations with the depression symptom composite $\left(r^{2}\right.$ range 21-28\%), these results demonstrate moderate associations between putatively depressogenic vulnerabilities with several types of anxiety symptoms. Regression analyses (Table 2) supplemented the correlational associations and revealed that approximately $24-28 \%$ of the variance in general anxiety and social phobia symptoms were accounted for by these putative vulnerabilities to depression. Notably, however, only $7-10 \%$ of the variance was accounted for in agoraphobic/interoceptive and specific fears. These results constitute an important extension of the literature because few if any studies have examined relationships between a wide range of anxiety symptoms with putatively depressogenic cognitive and personality-cognitive style vulnerabilities.

In addition, supplementary regression analyses demonstrated that the relationships between the vulnerabilities and anxiety symptoms were not entirely explained by the overlap of depression and anxiety. Thus, although most of the association between these vulnerabilities and anxiety outcomes lies in the overlap of depression and anxiety, there was also support found for some small associations independent of that overlap.

\section{Relationships Between Specific Vulnerabilities}

A second important finding was that, in general, shared rather than unique aspects of the four specific vulnerabilities were most important in accounting for symptom variance (e.g., Hankin et al. 2004). Although there were several instances in which certain vulnerabilities made small but statistically significant unique contributions to depression and anxiety symptoms, the vast majority of variance across outcomes was shared among the vulnerabilities. Specifically with regard to depression (composite and general depressive symptoms), social phobia, anxious arousal, agoraphobic/interoceptive, and general anxiety symptoms, approximately $70 \%$ of the variance accounted for by the specific vulnerabilities was shared across the specific vulnerabilities.

Incremental Validity of Specific Vulnerabilities

It is well know that $\mathrm{N}$ is related to both depression and anxiety (e.g., Clark et al. 1994; Watson et al. 2005). Further, it is a broad vulnerability factor and one that was significantly correlated with each of the four narrower vulnerabilities (see Table 1: $r$ 's range: .50-.58). The results of regression analyses revealed that neuroticism made the largest unique contributions to all eight outcomes. However, each of the specific vulnerabilities also made small but significant unique contributions to depressive symptoms and/or one or more types of anxiety symptoms.

The general pattern of results from this study is consistent with the findings of Zinbarg et al. (2010) who only examined past diagnoses but also found that the vast majority of variance accounted for by the vulnerabilities in depression and anxiety diagnoses was shared among the vulnerabilities. Indeed, for six of the eight outcomes reported in the present study, two-thirds of symptom variance was shared. Some preliminary analyses have been conducted to help elucidate the relationship between the overlap in the four specific vulnerabilities and N (Griffith et al. 2010b). These initial results suggest a very strong association $(>.8)$ between a latent variable derived from the four cognitive vulnerability measures and a latent variable derived from the multiple measures of $\mathrm{N}$ in our larger study.

Although $\mathrm{N}$ was a substantial predictor of all outcomes, our findings also demonstrate that the more narrow vulnerabilities provided small but significant incremental validity beyond $\mathrm{N}$. These results diverge from those of Zinbarg et al. (2010) who failed to find unique contributions for specific vulnerabilities beyond N. Importantly, our results suggest that with respect to current symptom levels of anxiety and depression, unlike past diagnoses, cognitive and personality-cognitive styles provide indices of risk that do not fully overlap with neuroticism. It remains to be determined in future prospective analyses whether the same pattern will hold.

\section{Limitations}

The present study should be viewed in light of several limitations. First, this investigation was cross-sectional and so no clear temporal ordering of predictors and outcomes can be claimed. Although affective state may influence the measurement of vulnerabilities, substantial trait (i.e., stable) variance has been demonstrated for many of these constructs, which suggests that these constructs are not just concomitants of distress but likely represent vulnerability factors for later psychopathology (e.g., Bagby et al. 2001; Hankin 2008b).

Second, the current study did not report on diagnostic outcomes. However, as noted above, symptom outcomes are important in their own right due to their associations with later disorders (e.g., Murphy et al. 1989; Pine et al. 1999). We also examined associations with a wide range of anxiety symptoms rather than lumping all anxiety disorders together and this provides an important complement to the results reported by Zinbarg et al. (2010). 
Third, although the theories about the vulnerabilities studied here are diathesis-stress in nature, only main effects were investigated. Cross-sectional methodology cannot adequately test diathesis-stress theories, which are more appropriately addressed with prospective designs. However, as noted by Zinbarg et al. (2010), diathesis-stress theories suggest an interaction of a form which is consistent with the finding of significant main effects for the vulnerabilities. Fourth, the present study investigated a sample of high-school juniors using only self-report measures. The results of the present study may not necessarily generalize to other populations. Further, some degree of overlap among the measures may be attributable to shared method variance.

Fifth, our community sample of adolescents was stratified on neuroticism upon study entry. Such a procedure could potentially limit the generalizability of the results or could have possibly inflated associations between neuroticism and outcomes. However, although high N participants were oversampled (59\%), medium and low $\mathrm{N}$ participants also participated in the study. Further, the study used a conservative measure of neuroticism by excluding items that overlapped with symptoms. Finally, initial simulations that we have conducted show that the level of oversampling used in this study should not have increased power more for $\mathrm{N}$ than for the other vulnerabilities (Hauner et al. 2010).

A final limitation is that, although this study assessed a wide range of anxiety symptoms, there were no symptom measures relevant to worry (GAD), obsessions/compulsions (OCD), or trauma symptoms (PTSD). Future studies might explore such associations; certainly $\mathrm{N}$ has demonstrated significant associations with those forms of anxiety (e.g., Watson et al. 2005).

\section{Conclusion}

In spite of these limitations, this study has several significant methodological strengths that allow some conclusions to be drawn about the relationships between $\mathrm{N}$, cognitive styles, personality-cognitive constructs, and symptoms of depression and anxiety. This study had a large, ethnically diverse sample of 550 adolescents, who completed a battery of psychometrically sound questionnaires. Symptoms were drawn from a range of instruments, and represented a wide sampling of depressive and anxiety symptomatology.

First, given the substantial relationship between symptoms of anxiety and dysfunctional attitudes, inferential style, sociotropy, and autonomy, future studies of these vulnerabilities should include measures of anxiety as well as depression. To the degree that substantial relationships exist between all of these vulnerabilities and particular types of anxiety, whether or not these associations are of lesser magnitude than their relationships with depression, the diatheses merit inclusion in predictive models of both anxiety and depression outcomes.

Second, future work on vulnerability constructs for depression should examine multiple constructs simultaneously to establish the incremental validity of different constructs. The present results highlighted that $\mathrm{N}$ had the most substantial unique associations with symptoms of depression as well as with symptoms of several types of anxiety. Although arguments have been raised against subsuming the more nuanced relationships between personality-cognitive styles and depression outcomes under $\mathrm{N}$ (see Zuroff et al. 2004b), parsimony also has value. Of the five vulnerabilities studied here, $\mathrm{N}$ offers the strongest predictor of current symptom levels and from an assessment standpoint is the most efficient vulnerability to measure.

Third, there may be some clinical utility to the finding that the specific vulnerabilities overlap with much of the association between $\mathrm{N}$ and symptoms, and that those vulnerabilities offer small, but significant, incremental validity. Whereas $\mathrm{N}$ is a broad variable, the specific measures of negative cognitive styles and personality orientations provide more fine-grained targets for both intervention and prevention. Indeed, studies focused on modifying negative cognitions have found preventive effects on depression in adolescents (e.g., Clarke et al. 2001).

Acknowledgments This research was supported by National Institute of Mental Health Grants R01 MH65651 to Richard Zinbarg and Susan Mineka (NU) and R01 MH65652 to Michelle Craske (UCLA). This research was also supported by National Institute of Mental Health Grant F31 MH076579 to the first author. Richard Zinbarg was also supported by the Patricia M. Nielsen Research Chair of the Family Institute at Northwestern University. Grateful acknowledgment is given to Jeff Jaeger, Angela Chiong, Lauren Spies, Catherine D’Avanzato, Corissa Callahan, and Natalie Castriotta.

Open Access This article is distributed under the terms of the Creative Commons Attribution Noncommercial License which permits any noncommercial use, distribution, and reproduction in any medium, provided the original author(s) and source are credited.

\section{References}

Alford, B. A., \& Gerrity, D. M. (1995). The specificity of sociotropyautonomy personality dimensions to depression vs. anxiety. Journal of Clinical Psychology, 51, 190-195.

Alloy, L. B., Abramson, L. Y., Hogan, M. E., Whitehouse, W. G., Rose, D. T., Robinson, M. S., et al. (2000). The TempleWisconsin cognitive vulnerability to depression project: Lifetime history of axis I psychopathology in individuals at high and low cognitive risk for depression. Journal of Abnormal Psychology, 109, 403-418.

Alloy, L. B., Abramson, L. Y., Whitehouse, W. G., Hogan, M. E., Panzarella, C., \& Rose, D. T. (2006). Prospective incidence of 
first onsets and recurrences of depression in individuals at high and low cognitive risk for depression. Journal of Abnormal Psychology, 115, 145-156.

Alloy, L. B., Kelly, K. A., Mineka, S., \& Clements, C. M. (1990). Comorbidity of anxiety and depressive disorders: A helplessness-hopelessness perspective. In J. D. Maser \& C. R. Cloninger (Eds.), Comorbidity of mood and anxiety disorders (pp. 499-543). Washington, DC: American Psychiatric Press.

Bagby, R. M., Gilchrist, E. J., Rector, N. A., Dickens, S. E., Joffe, R. T., Levitt, A., et al. (2001). The stability and validity of the sociotropy and autonomy personality dimensions as measured by the revised personal style inventory. Cognitive Therapy and Research, 25, 765-779.

Beck, A. T. (1967). Depression: Clinical, experimental, and theoretical aspects. New York: Harper and Row.

Beck, A. T., Epstein, N., Brown, G., \& Steer, R. A. (1988). An inventory for measuring clinical anxiety: Psychometric properties. Journal of Consulting and Clinical Psychology, 56, 893-897.

Clark, D. A., \& Beck, A. T. (with Alford, B. A.). (1999). Scientific foundations of cognitive theory and therapy for depression. New York: Wiley.

Clark, L. A., \& Watson, D. (1991). Tripartite model of anxiety and depression: Psychometric evidence and taxonomic implications. Journal of Abnormal Psychology, 100, 316-336.

Clark, L. A., Watson, D., \& Mineka, S. (1994). Temperament, personality, and the mood and anxiety disorders. Journal of Abnormal Psychology, 103, 103-116.

Clarke, G. N., Hornbrook, M., Lynch, F., Polen, M., Gale, J., Beardslee, W., et al. (2001). A randomized trial of a group cognitive intervention for preventing depression in adolescent offspring of depressed parents. Archives of General Psychiatry, $58,1127-1134$.

Costa, P. T., Jr., \& McCrae, R. R. (1985). The NEO personality inventory manual. Odessa, FL: Psychological Assessment Resources.

Costa, P. T., Jr., Terracciano, A., \& McCrae, R. R. (2001). Gender differences in personality traits across cultures: Robust and surprising findings. Journal of Personality and Social Psychology, 81, 322-331.

Dunkley, D. M., Blankstein, K. R., \& Flett, G. L. (1997). Specific cognitive-personality vulnerability styles in depression and the five-factor model of personality. Personality and Individual Differences, 23, 1041-1053.

Eysenck, H. J., \& Eysenck, S. B. G. (1975). Eysenck personality questionnaire manual. London: Hodder and Stoughton.

Eysenck, S. B. G., Eysenck, H. J., \& Barrett, P. (1985). A revised version of the psychoticism scale. Personality and Individual Differences, 6, 21-29.

Fergusson, D. M., Horwood, L. J., Ridder, E. M., \& Beautrais, A. L. (2005). Subthreshold depression in adolescence and mental health outcomes in adulthood. Archives of General Psychiatry, 62, 66-72.

Fresco, D. M., Sampson, W. S., Craighead, L. W., \& Koons, A. N. (2001). The relationship of sociotropy and autonomy to symptoms of depression and anxiety. Journal of Cognitive Psychotherapy: An International Quarterly, 15, 17-31.

Frewen, P. A., \& Dozois, D. J. A. (2006). Social, achievement, and control dimensions of personality-life event vulnerability to depression. Cognitive Therapy and Research, 30, 1-17.

Geer, J. H. (1965). The development of a scale to measure fear. Behavior Research and Therapy, 3, 45-53.

Goldberg, L. R. (1999). A broad-bandwidth, public domain, personality inventory measuring the lower-level facets of several fivefactor models. In I. Mervielde, I. Deary, F. De Fruyt, \& F. Ostendorf (Eds.), Personality psychology in Europe (Vol. 7, pp. 7-28). Tilburg, The Netherlands: Tilburg University Press.
Griffith, J. W., Zinbarg, R. E., Craske, M. G., Mineka, S., Rose, R. D., Waters, A. M., et al. (2010a). Neuroticism as a common dimension in the internalizing disorders. Psychological Medicine, 40, 1125-1136.

Griffith, J. W., Zinbarg, R. E., Mineka, S., Sutton, J. M., \& Craske, M. G. (2010b). Association of neuroticism and cognitive vulnerability for depression. Manuscript in preparation.

Haeffel, G. J., Gibb, B. E., Metalsky, G. I., Alloy, L. B., Abramson, L. Y., Hankin, B. L., et al. (2008). Measuring cognitive vulnerability to depression: Development and validation of the cognitive style questionnaire. Clinical Psychology Review, 28, 824-836.

Hankin, B. L. (2008a). Cognitive vulnerability-stress model of depression during adolescence: Investigating depressive symptom specificity in a multi-wave prospective study. Journal of Abnormal Child Psychology, 36, 999-1014.

Hankin, B. L. (2008b). Stability of cognitive vulnerabilities to depression: A short-term prospective multiwave study. Journal of Abnormal Psychology, 117, 324-333.

Hankin, B. L., Abramson, L. Y., Miller, N., \& Haeffel, G. J. (2004). Cognitive vulnerability-stress theories of depression: Examining affective specificity in the prediction of depression versus anxiety in three prospective studies. Cognitive Therapy and Research, 28, 309-345.

Hankin, B. L., Fraley, R. C., \& Abela, J. R. Z. (2005). Daily depression and cognitions about stress: Evidence for a traitlike depressogenic cognitive style and the prediction of depressive symptoms in a prospective daily diary study. Journal of Personality and Social Psychology, 88, 673-685.

Hauner, K., Zinbarg, R. E., \& Revelle, W. (2010). The effects of oversampling on directly and indirectly oversampled predictors. Manuscript in preparation.

International Personality Item Pool. (2001). A scientific collaboratory for the development of advanced measures of personality traits and other individual differences (http://ipip.ori.org/). Internet Web Site.

Klein, D. N., Durbin, C. E., \& Shankman, S. A. (2009). Personality and mood disorders. In I. H. Gotlib \& C. L. Hammen (Eds.), Handbook of depression (pp. 93-112). New York: Guilford Press.

Lewinsohn, P. M., Solomon, A., Seeley, J. R., \& Zeiss, A. (2000). Clinical implications of "subthreshold" depressive symptoms. Journal of Abnormal Psychology, 109, 345-351.

Mattick, R. P., \& Clarke, J. C. (1998). Development and validation of measures of social phobia scrutiny fear and social interaction anxiety. Behaviour Research and Therapy, 36, 455-470.

Mineka, S., Pury, C. L., \& Luten, A. G. (1995). Explanatory style in anxiety and depression. In G. M. Buchanan \& M. E. P. Seligman (Eds.), Explanatory style (pp. 135-158). Hillsdale, NJ: Lawrence Erlbaum Associates, Inc.

Mineka, S., Watson, D., \& Clark, L. A. (1998). Comorbidity of anxiety and unipolar mood disorders. Annual Review of Psychology, 49, 377-412.

Mongrain, M., \& Blackburn, S. (2005). Cognitive vulnerability, lifetime risk, and the recurrence of major depression in graduate students. Cognitive Therapy and Research, 29, 747-768.

Mor, N., Zinbarg, R. E., Craske, M. G., Mineka, S., Uliaszek, A., Rose, R., et al. (2008). Evaluating the invariance of the factor structure of the EPQ-R-N among adolescents. Journal of Personality Assessment, 90, 66-75.

Murphy, J. M., Sobol, A. M., Olivier, D. C., Monson, R. R., Leighton, A. H., \& Pratt, L. A. (1989). Prodromes of depression and anxiety: The Stirling County study. British Journal of Psychiatry, 155, 490-495.

Oliver, J. M., Murphy, S. L., Ferland, D. R., \& Ross, M. J. (2007). Contributions of the cognitive style questionnaire and the dysfunctional attitude scale to measuring cognitive vulnerability to depression. Cognitive Therapy and Research, 31, 51-69. 
Paunonen, S. V. (1998). Hierarchical organization of personality and prediction of behavior. Journal of Personality and Social Psychology, 74, 538-556.

Peterson, C., Semmel, A., von Baeyer, C., Abramson, L. Y., Metalsky, G. I., \& Seligman, M. E. P. (1982). The attributional style questionnaire. Cognitive Therapy and Research, 6, 287-300.

Pine, D. S., Cohen, E., Cohen, P., \& Brook, J. (1999). Adolescent depressive symptoms as predictors of adult depression: Moodiness or mood disorder? American Journal of Psychiatry, 156, 133-135.

Rapee, R., Craske, M., \& Barlow, D. H. (1995). An assessment instrument for panic disorder that includes fear of sensation producing activities. Anxiety, 1, 114-122.

Robins, C. J., Ladd, J., Welkowitz, J., Blaney, P. H., Diaz, R., \& Kutcher, G. (1994). The personal style inventory: Preliminary validation studies of new measures of sociotropy and autonomy. Journal of Psychopathology and Behavioral Assessment, 16, 277-300.

Tabachnick, B. G., \& Fidell, L. S. (2001). Using multivariate statistics. Boston: Allyn and Bacon.

Watson, D., Clark, L. A., Weber, K., Assenheimer, J. S., Strauss, M. E., \& McCormick, R. A. (1995a). Testing a tripartite model: II. Exploring the symptom structure of anxiety and depression in student, adult, and patient samples. Journal of Abnormal Psychology, 104, 15-25.

Watson, D., Gamez, W., \& Simms, L. J. (2005). Basic dimensions of temperament and their relation to anxiety and depression: A symptom-based perspective. Journal of Research in Personality, 39, 46-66.

Watson, D., Weber, K., Assenheimer, J. S., Clark, L. A., Strauss, M. E., \& McCormick, R. A. (1995b). Testing a tripartite model: I. Evaluating the convergent and discriminant validity of anxiety and depression symptom scales. Journal of Abnormal Psychology, 104, 3-14.

Weissman, A. N., \& Beck, A. T. (1978). Development and validation of the dysfunctional attitudes scale. Paper presented at the annual meeting of the Association for the Advancement of Behavior Therapy, Chicago.

Zimmerman, M., Coryell, W., Corenthal, C., \& Wilson, S. (1986). A self-report scale to diagnose major depressive disorder. Archives of General Psychiatry, 43, 1076-1081.

Zinbarg, R. E., \& Barlow, D. H. (1996). Structure of anxiety and the anxiety disorders: A hierarchical model. Journal of Abnormal Psychology, 105, 181-193.

Zinbarg, R. E., Mineka, S., Craske, M. G., Griffith, J. W., Sutton, J., Rose, R. D., et al. (2010). The northwestern-UCLA youth emotion project: Associations of cognitive vulnerabilities, neuroticism and gender with past diagnoses of emotional disorders in adolescents. Behaviour Research and Therapy, 48, 347-358.

Zinbarg, R., Yovel, I., Revelle, W., \& McDonald, R. (2006). Estimating generalizability to a universe of indicators that all have an attribute in common: A comparison of estimators for $\omega_{h}$. Applied Psychological Measurement, 30, 121-144.

Zuroff, D. C. (1994). Depressive personality styles and the five-factor model of personality. Journal of Personality Assessment, 63, 453-472.

Zuroff, D. C., Mongrain, M., \& Santor, D. A. (2004a). Conceptualizing and measuring personality vulnerability to depression: Comment on Coyne and Whiffen (1995). Psychological Bulletin, 130, 489-511.

Zuroff, D. C., Mongrain, M., \& Santor, D. A. (2004b). Investing in the personality vulnerability research program-Current dividends and future growth: Rejoinder to Coyne, Thompson, and Whiffen (2004). Psychological Bulletin, 130, 518-522. 